\title{
Massimo Campanini (1954-2020)
}

Massimo Campanini è stato un intellettuale dal multiforme ingegno con una produzione scientifica e divulgativa amplissima ed estremamente variegata: i suoi interessi andavano dalla filosofia alla storia, dal pensiero politico all'islamistica. Ha sempre avuto un approccio naturalmente interdisciplinare integrando nelle sue ricerche lo sguardo del filosofo, l'accuratezza del linguista e l'analisi dello storico.

Lo sguardo aperto e curioso che lo caratterizzava, così come l'impeto e la passione con cui si cimentava nei campi di studio più diversi, rappresentano delle caratteristiche ormai rare nel panorama culturale contemporaneo, dove spesso la tendenza a chiudersi in settori disciplinari sempre più angusti è la cifra comune della maggior parte dell'accademia.

Se appare difficile tracciare un quadro unitario della sua produzione e del suo pensiero può essere utile ripercorrere le tappe della sua formazione e tornare alle origini, ossia allo studio della filosofia presso l'Università degli Studi di Milano. Questa prima affiliazione si rivela infatti fondamentale perché ha condizionato tutto il suo successivo percorso fornendogli una chiave di lettura storico-critica della tradizione filosofica, prospettiva che non ha mai abbandonato e che si ritrova nei suoi studi di filosofia islamica. Campanini si è laureato nel 1977 con una tesi sul concetto di infinito in Giordano Bruno, in quello stesso dipartimento dove ha insegnato Mario Dal Pra (dall'inizio degli anni Cinquanta) che con le sue ricerche e la formulazione del trascendentalismo della prassi ha avviato un modo originale di pensare la storia della filosofia.

Il suo accidentato percorso accademico, iniziato tardivamente quando ormai era uno studioso affermato e conosciuto, lo ha visto per diversi anni tenere un insegnamento di Cultura Araba presso l'Università degli Studi di Milano, solo nel 2005 ha ottenuto un posto da ricercatore presso l'Università Orientale di Napoli dove ha insegnato Storia contemporanea dei paesi arabi e Storia dell'Islam Contemporaneo, insegnamento che lo ha indirizzato verso l'approfondimento degli studi storici e, infine, ha concluso il suo percorso come professore associato presso l'Università degli Studi di Trento (dal 2011 al 2016), dove insegnava Pensiero Islamico e Storia dei Paesi Islamici. Dal 2016 era anche accademico della prestigiosa Accademia Ambrosiana di Milano per la Classis Orientalis di arabo, ma le sue affiliazioni sono davvero numerose, sia a prestigiose istituzioni nazionali e internazionali che nei comitati di redazione di importanti riviste.

La sue ricerche si sono concentrate all'inizio sullo studio della filosofia in età medievale; le traduzioni dei testi di Averroè e al-Fārābī (tra cui di Averroè Il Trattato decisivo, Rizzoli 1994, L'incoerenza dell'Incoerenza dei filosofi, U.T.E.T. 1997; di al- Fārābī La città Virtuosa, Rizzoli 1996), con gli studi che le accompagnano, hanno reso disponibili ai lettori italiani non specialisti i testi di questi grandi pensatori che hanno giocato un ruolo fondamentale nel passaggio della tradizione aristotelica all'occidente latino; si tratta 
inoltre di autori la cui riflessione in campo metafisico, politico e gnoseologico ha avuto un grandissimo impatto sul pensiero occidentale, che in effetti ne ha raccolto in modo diretto l'eredità.

Riprendendo l'idea di Alain De Libera di un medioevo plurale Campanini combatteva le diverse forme di «oblio» dell'Islam in Occidente, cioè, «quell'atteggiamento per cui la cultura europea ha sistematicamente e scientemente, negato e respinto i legami che la stringevano all'Islam» (dall'Introduzione a Dante e L'islam, Studium Edizioni 2019). Il suo approccio teorico profondamente anti-orientalista, come lui stesso lo definiva, e il tentativo di dare una lettura organica della tradizione filosofica islamica sono tratti peculiari dei suoi studi in questo ambito.

Campanini ha infatti proposto una lettura originale della storia della filosofia islamica, inserendo autori che non sono strettamente dei filosofi come al-Ghazālì, alMāwardī, Ibn Taymiyya, Ibn Khaldūn, per citare i nomi più noti. Si tratta di pensatori la cui produzione ha avuto una eco considerevole nella cultura arabo-islamica; d'altra parte nemmeno il più famoso Averroè, a cui ha dedicato diversi contributi (si ricorda qui Averroè, Il Mulino 2007), si può considerare semplicemente un filosofo ma un intellettuale militante, un intellettuale nel senso gramsciano del termine, in quanto si faceva portavoce di un progetto politico (quello almohade). Molti di questi autori, sottolineava Campanini, erano uomini pienamente inseriti nel loro tempo storico, protagonisti di primo piano delle vicende politiche e vissuti, nella maggior parte dei casi, a stretto contatto con il Potere; per questo egli respingeva con forza la tesi di Leo Strauss che individuava un doppio registro linguistico e comunicativo dei filosofi musulmani. Per Campanini, sempre sensibile a indagare le categorie del "politico» nella storia del pensiero, esisteva una relazione dialettica tra i filosofi e la città, un rapporto di coinvolgimento ed esclusione; e tuttavia questo non poteva tradursi nell'idea che i filosofi fossero costretti a nascondere le loro dottrine dietro una patina di conformismo e ortodossia religiosa.

Proprio la definizione di un campo di studio così ampio ha permesso a Campanini di trattare in modo approfondito, accanto ai tradizionali commentatori di Aristotele (i più noti falasifa), alcuni importanti teologi come al-Ghazālī (a cui ha dedicato molti studi tra cui al-Ghazāli and the Divine, Routledge 2018) e Ibn Taymiyya, storici come al-Māwardī e Ibn Khaldūn (Ibn Khaldun e la Muqaddima, La Vela 2019; La politica nell'Islam. Una Interpretazione, Il Mulino 2019), mentre ha dedicato meno attenzione al filone avicenniano, distaccandosi in modo deciso dalla scuola storiografica legata a Henry Corbin.

Tra i temi che attraversano la sua produzione in modo trasversale ricordiamo il problema della dialettica tra fede e ragione (o tra religione e filosofia): ripresa dal mondo latino e dai cosiddetti averroisti rappresenta in realtà un falso problema per gli autori arabo-islamici, dato che i due ambiti o si consideravano come assolutamente divaricati e irriducibili o si riteneva che vi fosse pacifica convivenza nel momento in cui interagivano. Certo esisteva la distinzione tra le cosiddette discipline razionali ('ulüm naqliyya) e quelle tradizionali ('ulūm 'aqliyya) ma la scienza teologica del kalām, scienza della Tradizione per eccellenza, non era affatto aliena alla logica e alle argomentazioni filosofiche, così come 
un giurista e teologo tradizionalista come Ibn Taymiyya, pur condannando la logica dei filosofi, era molto attento al rigore argomentativo. Anche le letture in chiave iperrazionalista di Averroè (così come quelle nettamente modernizzanti di Ibn Khaldūn) sono frutto di una visione che distacca il filosofo e le sue opere dall'ambiente in cui era pienamente inserito. Scrive a proposito Campanini: «E tutto questo è perfettamente coerente con il progetto politico almohade (almeno come interpretato da Averroè): proteggere la scienza, propagandare la fede presso le masse ed emarginare i teologi e i giuristi conservatori che pretendono di dettare al califfo la sua politica. Su questa prospettiva gnoseologico-politica ruota l'ultima parte del Trattato Decisivo» (Averroè, cit. p. 71)

D'altra parte autori come Averroè e Ibn Khaldūn sono dei simboli e rappresentano delle fratture nella coscienza storica dell'Islam: non sono interessanti solo per le loro opere e dottrine ma anche per le letture che ne sono state date nel corso degli anni. In particolare nel mondo arabo-islamico contemporaneo la ricerca di un filone di razionalismo autoctono, per venire a patti con la modernità, ha condizionato la storiografia filosofica e le interpretazioni di autori come Muhammad al-Jābrī, 'Abdelwahab Bouhdiba e altri. Così Averroè (e per certi versi Ibn Khaldūn) ha subito un singolare destino nel corso del tempo: senza epigoni nel mondo arabo ma con un grande seguito in quello latino occidentale, viene letteralmente riscoperto dal pensiero arabo contemporaneo alla ricerca di padri nobili a cui fare riferimento in un cammino di riscatto ed emancipazione dall'orientalismo europeo.

Questi corsi e ricorsi della storia dimostrano, come sottolineava Campanini, che quelle islamica e latino-occidentale sono civiltà profondamente interdipendenti che hanno continuato a dialogare e interagire nel corso dei secoli; la vocazione profondamente mediterranea dell'Islam ne fa a pieno titolo una religione dell'occidente, erede anch'essa della cultura greca: «l'Islam ha condiviso e condivide con l'Europa, l'ebraismo e il cristianesimo innanzi tutto le medesime radici abramitiche della religione e i medesimi paradigmi culturali (tra cui il sostrato filosofico greco e tardoantico) e in seguito tutto il tessuto di corrispondenze e di interscambi commerciali, linguistici e soprattutto etico-comportamentali che contraddistinguono le civiltà mediterranee» (Campanini, Dante e L'Islam, cit., p. 12)

Un altro leitmotiv che si trova in molte opere di Campanini è l'analisi del concetto di stato islamico e la dialettica tra utopia e antiutopia, tema che ha trattato sia nei testi dedicati al medioevo sia in quelli sul pensiero contemporaneo. Nel suo celebre studio Islam e Politica (Il Mulino 1999 e successive riedizioni), punto di svolta della sua ricerca, affronta il tema facendo una disamina storica dall'epoca classica fino al XX secolo. Se i filosofi della tradizione medievale come al-Fārābī, Averroè, Avempace, e un autore classico come alMāwardī (XI sec.), parlavano dello stato islamico (e del califfato) in termini ideali, parallelamente si era evoluto un pensiero ancorato alla realtà storica delle istituzioni islamiche, realtà in cui il califfato e lo stato islamico nella sua forma ideale non esisteva più e non avrebbe mai potuto realizzarsi una seconda volta dopo l'epoca perfetta di 
Muhammad e dei ben guidati. Si tratta dell'atteggiamento che Campanini definiva di utopia retrospettiva per cui ai posteri non restava che elaborare un modello islamico di stato (dato che l'ideale del califfato non poteva più realizzarsi), in cui l'utopia non si collocava nel futuro ma nel passato a cui cercare di ritornare; accanto a questo atteggiamento che avrà grande influenza sul pensiero contemporaneo e sulle correnti salafite, si collocavano il quietismo di al-Ghazālī che vedeva nell'interiorità dell'uomo il campo su cui combattere il jihād più importante, e il realismo di Ibn Khaldūn, coscienza critica dell'Islam al declinare dell'età classica.

Alcuni elementi paiono in conclusione imprescindibili per descrivere l'atteggiamento teorico di Campanini: l'approccio profondamente anti-orientalista, il tentativo di proporre una lettura «organica» della tradizione islamica che metteva in evidenza l'esistenza di un pensiero filosofico con radici autoctone e non semplicemente il canale di trasmissione dell'eredità greca all'occidente latino, infine il costante riferimento ai testi e alle fonti primarie di cui aveva una profonda conoscenza e una ininterrotta frequentazione.

Accanto agli studi più strettamente filosofici Campanini ha esplorato il pensiero contemporaneo, gli studi storici e non ha mai smesso la ricerca sul testo del Corano e sul profeta Muhammad. Anche in tali testi è possibile ritrovare le domande che hanno accompagnato tutta la sua ricerca (ancora una volta il tema del politico) e uno sguardo aperto sui possibili scenari futuri della cultura e della civiltà islamica. Gli studi sull'ermeneutica coranica (L'esegesi musulmana del Corano nel secolo Ventesimo, Morcelliana 2008 e Philophical Perspectives on Modern Qur'anic Exegesis. Key Paradigms and Concepts, Equinox Publishing 2016) così come la biografia di Muhammad (Maometto, Salerno Editore 2020) ultima e importante fatica pubblicata prima della sua morte improvvisa, completano, senza esaurirlo, il quadro di questo studioso.

Massimo è stato un maestro generoso e un intellettuale militante, nel senso gramsciano, non ha mai rinunciato al confronto con il mondo e con la storia. Nell'ottobre del 2020 è mancato, una lunga malattia lo aveva costretto a ridurre viaggi e spostamenti ma la perdita è stata improvvisa e dolorosa. I semi che ha coltivato amorevolmente attraverso il tempo dedicato ai suoi tanti allievi continueranno a far vivere la sua lezione.

Francesca Forte

Investigadora independiente 